\title{
Revistas latinoamericanas de ciencia, tecnología y medicina en la base de datos Periódica: una visión de 40 años
}

\author{
Latin-American journals of Science, Technology and Medicine in Periodica database: a 40-year overview
}

\author{
José Octavio Alonso-GamboA, Felipe Rafael ReYnA-EsPinosa \\ Universidad Nacional Autónoma de México, Dirección General de Bibliotecas, Ciudad de México, \\ oalonso@unam.mx,frre@unam.mx
}

\begin{abstract}
Resumen
Se analizan las características más sobresalientes de un conjunto de revistas latinoamericanas especializadas en ciencia, tecnología y medicina (CTM), a partir de los documentos compilados desde 1978 hasta 2018 en la base de datos Periódica. En ocasión del 40 aniversario de esta base de datos se hace también referencia a su situación actual y se ofrece una reflexión sobre las aportaciones de las bases de datos bibliográficas.
\end{abstract}

Palabras clave: Revistas académicas. Bases de datos bibliográficas. América Latina. Medicina. Ciencia. Tecnología. Periódica. México.

\section{Introducción}

En la década de los años setenta, la Universidad Nacional Autónoma de México (UNAM) se dio a la tarea de crear productos de información con dos objetivos principales: difundir registros bibliográficos a partir del análisis de documentos publicados en revistas científicas latinoamericanas y contribuir al desarrollo de servicios de información especializados en revistas de América Latina, producto por demás poco conocido y disperso. La creación de estos productos de información también respondía a la escasa representatividad de revistas de la región en los servicios internacionales especializados (Alonso, 1998; Michán, 2008).

Es en este contexto donde en 1978 nace la base de datos Periódica (Índice de Revistas Latinoamericanas en Ciencias), como un servicio de información dedicado a la compilación, organización, acceso y preservación de documentos publicados en revistas académicas de América Latina y el Caribe, especializadas en ciencia, tecnología y medicina (CTM, en adelante). Con la creación de Periódica, la UNAM complementó la difusión de revistas latinoamericanas ya que tres años antes había iniciado la producción de la

\begin{abstract}
The most outstanding characteristics of the literature published in Latin American journals specialized in Science, Technology and Medicine (STM) are described, from documents compiled from 1978 to 2018 in the Periódica database. On the 40th anniversary of this database, reference is made to its current situation and future challenges as well as a reflection on the contributions of bibliographic databases.
\end{abstract}

Keywords: Scholarly journals. Bibliographical databases. Latin America. Medicine. Science. Technology. Periodica. Mexico.

base de datos CLASE, especializada en revistas de ciencias sociales y humanidades (Reyna, 2015).

Ambas bases de datos fueron creadas en el Centro de Información Científica y Humanística $(\mathrm{ClCH})$, y actualmente se producen en la Dirección General de Bibliotecas (DGB) de la Universidad Nacional Autónoma de México (UNAM). Los antecedentes de creación de Periódica están detallados en un artículo publicado en 1983 (Maffey, 1983), mismo que sirve de punto de partida a este trabajo.

Cuando Periódica fue creada, la difusión de sus registros se daba a través de publicaciones impresas de aparición trimestral, las cuales se mantuvieron como el principal medio de difusión durante sus primeros 20 años (1978-1998), período en el que también se difundieron mediante acceso remoto (entre 1986 y 1993) y en soporte CDROM (entre 1989 y 1997). No fue sino hasta abril de 1998 cuando los contenidos de Periódica (y también los de CLASE) estuvieron disponibles vía Internet (Alonso, 1998), forma de acceso que se mantiene hasta hoy día. Periódica puede ser consultada en periodica.unam.mx y la oficina técnica encargada de su producción es el Departamento 
de Bibliografía Latinoamericana de la DGB, donde se desarrollan también otros servicios de información sobre revistas latinoamericanas: CLASE (clase.unam.mx), Latindex (www.latindex.org), SciELO-México (www.scielo.org.mx) y el portal Biblat (biblat.unam.mx), que ofrece acceso al conjunto completo de referencias bibliográficas de CLASE y Periódica, así como frecuencias e indicadores bibliométricos extraídos del conjunto de revistas de ambas bases de datos.

En sus inicios Periódica intentó cubrir de la manera más amplia todos los materiales considerados relevantes para la base de datos, incluyendo varios boletines y manuales. Con el paso del tiempo muchos de estos materiales desaparecieron para dar lugar a revistas más formales. A finales de la década de los noventa la selección de las publicaciones a ser indexadas quedó en manos de un comité de selección y se publicaron los primeros criterios, basados en los construidos por Latindex, con lo cual el universo a cubrir fue haciéndose más selectivo (Alonso, 1998).

Un grupo de documentalistas especializados crea un registro bibliográfico de cada documento (artículos originales y de revisión, comunicaciones cortas, informes técnicos, estudios de caso y otros), con base en la lectura y análisis del texto completo. Actualmente cada registro se compone de 20 metadatos entre los que se encuentran: título de la revista, título del documento, nombre de los autores, afiliaciones de trabajo de los autores, disciplinas, idioma del documento, idioma de los resúmenes, los resúmenes hasta en tres idiomas, palabras clave en español, keywords, referencias, tipo de documento y enfoque del mismo. A raíz de la publicación electrónica se incluyen además datos como el enlace al texto completo y el número DOI de los documentos.

Por su cobertura regional, la base de datos Periódica tiene las características para ofrecer una nueva perspectiva de la ciencia que se realiza en la región ya que integra revistas que no son consideradas en otros servicios de información trasnacionales, que además tienen un perfil comercial (Chong et al., 2018).

En este trabajo se presentan los resultados del análisis de las principales características de las revistas de CTM indexadas en Periódica, así como de los documentos publicados, a partir de los datos compilados durante 40 años por la base de datos.

\section{Metodología}

Lo primero que se realizó fue verificar la vigencia de las publicaciones a partir de un listado oficial de publicaciones originado en el Departamento de Bibliografía Latinoamericana. Se realizó un vaciado de los títulos que contenían algún registro en la base de datos con información sobre el país de edición, ISSN, vigencia, temática, soporte y periodicidad. Para la clasificación por temas se utilizó la propia de Periódica documentada en su manual de indexación (Alonso, 2012). Para comparar el porcentaje de cobertura de $\mathrm{Pe}$ riódica del total de revistas de CTM en América Latina se contrastaron los datos con los del Catálogo Latindex, delimitado para revistas de América Latina, vigentes y de las disciplinas cubiertas por Periódica.

A nivel de documento se analizaron dos indicadores revisados también el artículo de 1983: el idioma de publicación y el país de adscripción de los autores. En estos casos la fuente de información fue la lista de control del sistema automatizado de referencias bibliográficas Aleph que se utiliza para el ingreso de datos y cuyo acceso está restringido a los documentalistas. Toda la información fue exportada a un archivo de Excel® para su análisis.

\section{Resultados}

\subsection{Revistas indexadas}

El primer número publicado de Periódica en 1978 cubrió un total de 334 revistas, 57 de ellas de México $(17 \%)$ que era el país mejor representado. Para abril de 2018, en la base de datos se analizaba el contenido de 1,162 revistas vigentes (tabla 1), en donde las revistas brasileñas son mayoría con casi un $30 \%$. Entre 1978 y 2018 la cobertura de títulos únicos de Periódica creció 3.5 veces.

Históricamente la base de datos ha recogido los contenidos de 1,593 revistas a partir de las cuales se han originado poco más de 415,000 registros bibliográficos (abril de 2018). Del total de revistas, 431 han dejado de publicarse, es decir, el $27 \%$, lo que refleja la inestable pervivencia de algunas revistas en la región, sobre todo en los primeros años de la colección. Aunque 20 países de la región cuentan con alguna revista indexada en $\mathrm{Pe}$ riódica, destaca el predominio de revistas brasileñas y mexicanas que en conjunto representan el $53 \%$, seguida de revistas de Argentina, Colombia y Cuba en ese orden. Además se registran 12 títulos editados por organismos internacionales. 


\begin{tabular}{lr}
\hline País & Revistas \\
\hline Argentina & 104 \\
\hline Belice & 1 \\
\hline Bolivia & 6 \\
\hline Brasil & 343 \\
\hline Chile & 66 \\
\hline Colombia & 117 \\
\hline Costa Rica & 24 \\
\hline Cuba & 85 \\
\hline República Dominicana & 2 \\
\hline Ecuador & 16 \\
\hline Guatemala & 1 \\
\hline Honduras & 2 \\
\hline México & 264 \\
\hline Nicaragua & 3 \\
\hline Organismos internacionales & 12 \\
\hline Panamá & 3 \\
\hline Paraguay & 1 \\
\hline Perú & 25 \\
\hline Puerto Rico & 1 \\
\hline Uruguay & 78 \\
\hline Venezuela &
\end{tabular}

Tabla 1. Revistas vigentes indexadas en Periódica, por país

\begin{tabular}{lr}
\hline Disciplina & Revistas \\
\hline Medicina & 407 \\
\hline Biología & 229 \\
\hline Ingeniería & 115 \\
\hline Agrociencias & 109 \\
\hline Geociencias & 71 \\
\hline Multidisciplinarias & 59 \\
\hline Ciencia y tecnología & 50 \\
\hline Química & 48 \\
\hline Medicina veterinaria y zootecnia & 23 \\
\hline Matemáticas & 20 \\
\hline Física y astronomía & 19 \\
\hline Ciencias de la computación & 12 \\
\hline
\end{tabular}

Tabla 2. Revistas vigentes indexadas en Periódica, por disciplina

\subsection{Revistas por disciplina}

La representación por disciplinas del conocimiento evidencia la primacía de las revistas de medicina $(407=35 \%)$ y biología $(229=20 \%)$ que en conjunto representan más de la mitad de toda la colección indexada. Las revistas de ingeniería, agrociencias y geociencias les siguen en número. Estos datos coinciden con los informados en el artículo publicado en 1983: medicina (30\%), biología (22\%) y agrociencias (17\%). Las revistas de física, astronomía y matemáticas que en ese entonces eran 15 , han aumentado ahora su número a 39.

Para tener una idea de qué tanto cubre Periódica el universo de títulos latinoamericanos de CTM, se realizó una búsqueda en el Catálogo Latindex para conocer el número de revistas vigentes en esas disciplinas para los países de América Latina. El conjunto de búsqueda se restringió al Catálogo ya que este arroja números referidos a revistas que han cumplido criterios de calidad muy similares a los que utiliza Periódica. El Catálogo Latindex arrojó un total de 2,123 revistas de CTM vigentes, por lo que Periódica cubre el $55 \%$ de dicho total.

\subsection{Revistas por institución editora}

Al analizar el tipo de institución editora de la revista, se confirma la amplia contribución de las universidades y asociaciones científicas en la edición de revistas latinoamericanas tal como se ha evidenciado en otros estudios (Cetto y Alonso, 1998). En conjunto, las instituciones educativas y las asociaciones profesionales son responsables editoriales del $70 \%$ del total de títulos, con una escasa participación de las editoriales comerciales con un $6 \%$ del total.

\begin{tabular}{lr}
\hline Tipo de institución editora & Revistas \\
\hline $\begin{array}{l}\text { Instituciones educativas (incluye } \\
\text { universidades) }\end{array}$ & 483 \\
\hline Asociaciones científicas y profesionales & 325 \\
\hline Instituciones gubernamentales & 135 \\
\hline $\begin{array}{l}\text { Instituciones de investigación (no incluye } \\
\text { universidades) }\end{array}$ & 117 \\
\hline Editoriales privadas, comerciales & 73 \\
\hline Organismos internacionales & 29 \\
\hline
\end{tabular}

Tabla 3. Revistas vigentes indexadas en Periódica, por tipo de institución

\subsection{Revistas por frecuencia de publicación}

Las periodicidades menores a semestral (mensual, bimestral, trimestral y cuatrimestral) consti- 
tuyen ya más de la mitad de la colección ( $56 \%=$ 656 revistas) lo cual en principio resulta favorable para mantener un buen ritmo de actualización de los contenidos. En el artículo de 1983 se mencionaba que el $33.8 \%$ de las publicaciones eran de aparición irregular (Maffey, 1983) situación que parece haber cambiado notablemente ya que para 2018 solamente el $9 \%$ de las publicaciones (105 revistas) se reportan bajo tal situación. El número de publicaciones con periodicidades muy extendidas es minoritario, ya que las publicaciones anuales o bienales sólo representan el $7 \%$.

\begin{tabular}{lr}
\hline Frecuencia de publicación & Revistas \\
\hline Trimestral & 312 \\
\hline Semestral & 300 \\
\hline Cuatrimestral & 175 \\
\hline Bimestral & 125 \\
\hline Irregular & 105 \\
\hline Anual & 79 \\
\hline Mensual & 44 \\
\hline Otras frecuencias & 19 \\
\hline Bienal & 3 \\
\hline
\end{tabular}

Tabla 4. Revistas por periodicidad

\subsection{Revistas por soporte}

La aparición de las primeras revistas latinoamericanas en línea, en la década de los noventa, facilitó la identificación y acceso a sus contenidos, reforzada además por su consulta libre y gratuita.

Actualmente, un $46 \%$ de los títulos (537) corresponden a revistas impresas que tienen una versión en línea, a las que se agregan 132 títulos $(11 \%)$ de revistas nacidas totalmente electrónicas. Si se suman los dos conjuntos puede decirse que más de la mitad de la colección (57\%) corresponde a publicaciones cuyos textos completos pueden ser consultados en línea. El cambio a la publicación electrónica de revistas, impulsó en 2004 la decisión de ingresar enlaces electrónicos en cada registro de Periódica para facilitar a los usuarios la consulta a los textos completos. Para abril de 2018 un total de 157,000 registros (38\%) contenían al menos una dirección de enlace.

No obstante, las revistas impresas siguen siendo importantes en la región; para paliar las dificultades que representa el alto costo de la mensajería, se han establecido acuerdos con los editores de las revistas impresas para que faciliten una versión PDF de los artículos, mismos que han sido integrados en un repositorio denominado HEVILA (Hemeroteca Virtual Latinoamericana), el cual contiene ya más de 12,000 artículos en dicho formato. Los enlaces a HEVILA están también disponibles desde cada registro lo que favorece la consulta a textos completos de publicaciones que todavía se distribuyen exclusivamente en papel.

\begin{tabular}{lr}
\hline Soporte de edición & Revistas \\
\hline Impresas + en línea & 537 \\
\hline Solamente impresas & 493 \\
\hline Solamente en línea & 132 \\
\hline
\end{tabular}

Tabla 5. Revistas vigentes indexadas en Periódica, por soporte de publicación.

\subsection{Artículos según idioma de publicación}

$\mathrm{Al}$ analizar el idioma en el que están escritos los documentos de las revistas de CTM latinoamericanas, se evidencia el uso predominante de tres idiomas: el portugués, el inglés y el español que en conjunto representan casi la totalidad (97\%) de los 236,905 registros de Periódica que contienen este dato. Llama la atención la primacía del portugués con el $37 \%$, seguido del inglés con el $31 \%$, mientras que al español corresponde un $29 \%$. En 1983 los resultados fueron muy diferentes ya que en ese entonces el español y el portugués eran los idiomas predominantes, mientras que el inglés lo era solamente para las revistas de física. Otros estudios basados en Periódica muestran el predominio del español como es el caso de los artículos de taxonomía o sistemática (Michan, 2008), usado en un $75 \%$ de los documentos analizados.

\begin{tabular}{lr}
\hline Idioma & Artículos \\
\hline Portugués & 88,045 \\
\hline Inglés & 72,824 \\
\hline Español & 68,734 \\
\hline Español e inglés & 4,366 \\
\hline Portugués e inglés & 2,460 \\
\hline Español, portugués e inglés & 317 \\
\hline Francés & 143 \\
\hline Alemán & 10 \\
\hline Italiano & 5 \\
\hline Español, portugués, inglés y francés & 1 \\
\hline Total & 236,905 \\
\hline
\end{tabular}

Tabla 6. Idioma de los artículos indexados

\subsection{Procedencia geográfica de los autores}

Periódica, al igual que su base de datos hermana CLASE, ha recogido desde su inicio información 
sobre la adscripción de los autores. El asentamiento de la adscripción, únicamente del primer autor, subsistió hasta el año 2009, cuando se decidió ingresar las adscripciones de todos los autores. Las adscripciones recogen los datos de la institución de trabajo de cada autor, así como información sobre la ciudad y el país, de tal modo que pueden obtenerse frecuencias por autor, institución, ciudad y país de afiliación. Estos datos permiten también evidenciar qué tan internacionales resultan ser nuestras revistas para lo cual se hizo una cuantificación por país de procedencia; los 10 países mejor representados se muestran en la tabla 7.

\begin{tabular}{lr}
\hline País & Adscripciones \\
\hline México & 88,109 \\
\hline Brasil & 61,385 \\
\hline Colombia & 20,908 \\
\hline Argentina & 16,226 \\
\hline Estados Unidos & 11,649 \\
\hline Chile & 11,391 \\
\hline España & 11,340 \\
\hline Venezuela & 11,074 \\
\hline Cuba & 6,165 \\
\hline Costa Rica & 3,959 \\
\hline
\end{tabular}

Tabla 7. País de la institución de adscripción de los autores

\section{Panorama de las bases de datos bibliográficas}

El aniversario de Periódica, es una excelente oportunidad para reflexionar y recoger la experiencia de un producto de información latinoamericano de largo aliento, y aprovechar para plasmar reflexiones y experiencias respecto a estos servicios de información.

En cuatro décadas de existencia, Periódica ha sido testigo del devenir de las revistas académicas y de las bases de datos que las difunden. De un inicio lento en los años setenta, la creación de bases de datos se aceleró en la década de los ochenta al grado de que hubo la necesidad de editar el Directorio DIBALC sobre la existencia de estos recursos en América Latina y el Caribe. A partir de aquí se dio la sobrevivencia sólo de aquellas bases que lograron adaptarse a los cambios tecnológicos, mantener sus objetivos, ritmo de trabajo y apoyo institucional. Una de las escasas sobrevivientes de este proceso es $\mathrm{Pe}$ riódica.
Los principales cambios que se han identificado en el campo de las revistas académicas que difunde Periódica, son las referentes a la mejora en criterios de calidad, gracias al trabajo de Latindex $y$ otras iniciativas regionales, el perfeccionamiento de las tecnologías de la información y comunicación que permitieron hacer más rápidos los procesos editoriales, así como la aparición y desarrollo de la publicación electrónica que facilitó la mejora de procesos de gestión editorial, acceso, preservación y uso de la información.

Aunado a esta evolución, la aparición de la revista electrónica en 1987, infundió una nueva dinámica a las bases de datos que eventualmente pudieron guiar al usuario hasta el texto completo de los documentos. Asimismo, los adelantos en la tecnología de cómputo y telecomunicaciones que dieron origen a la aparición de Internet, hicieron posible a las revistas y bases de datos romper las barreras físicas que les constreñían logrando tener la ansiada difusión internacional.

La aparición de los buscadores de información tipo Google tuvo gran impacto en la comunidad de usuarios debido a la abundante cantidad de resultados que arrojaban las consultas. Sin embargo, con la explosión de la información y la abundancia de fuentes de información no confiables, los usuarios académicos se dieron cuenta que seguían siendo necesarias herramientas selectivas y controladas como las bases de datos bibliográficas. Tal como lo señalan Giménez y Rodríguez (2012), las bases de datos bibliográficas aportan estructura a búsquedas de información más elaboradas; están respaldadas por estrategias de búsqueda, control de vocabularios, análisis documental y selección de fuentes, que proporcionan resultados más exhaustivos y certeros.

Buscando aportar innovaciones a los usuarios, en 2009 fue creado el portal BIBLAT [https://biblat.unam.mx/es] que fusionó los registros tanto de CLASE como de Periódica, potenciando no sólo la cantidad de documentos disponibles (poco más de 880,000 ), sino integrando funcionalidades de interés para los estudiosos de la bibliometría en la región. El objetivo fue ofrecer un producto más robusto que, además de las tradicionales funciones de búsqueda y recuperación de información, permiten conocer indicadores a través del análisis de frecuencias y la generación de indicadores bibliométricos de las revistas y documentos analizados por CLASE y Periódica. En este sentido, Biblat representa una evolución y una reutilización de los recursos de dos bases de datos con información académica procesada por más de 40 años, para darle nuevos usos.

Biblat permitió además aumentar la difusión y el uso de ambas bases de datos. Lo anterior se 
refleja en los informes generados por Google Analytics que indican que de 2014 a marzo de 2018 , las consultas acumuladas sumaron casi 4 millones de usuarios, con un promedio de 4,000 consultas por día. El $78 \%$ de las consultas provienen de cinco países: México $(1,671,750 /$ $42 \%)$, China $(806,503 / 20 \%)$, Colombia $(272,022 /$ $7 \%)$, Brasil $(196,854 / 5 \%)$ y Perú $(148,415 / 4 \%)$.

La modernización de los servicios y funciones de las bases de datos bibliográficas también se ve reflejada en acciones como la recientemente anunciada (junio de 2018) por el Consejo Superior de Investigaciones Científicas de España (CSIC), al crear un nuevo producto denominado ÍnDICEs-CSIC que integrará los acervos de ICYT (Ciencia y Tecnología), IME (Biomedicina) e ISOC (Ciencias Sociales y Humanidades), con el objetivo de ofrecer productos más robustos a los usuarios.

En el mundo desarrollado la industria de las bases de datos bibliográficas continúa siendo importante, pero ahora la gran mayoría de ellas ofrecen además el acceso a textos completos mediante enlaces incluidos en sus registros. De acuerdo con la página web de Gale Cengage, compañía productora del Directory of Online Databases, en la edición 40 , existen casi 14,000 bases de datos en línea, más del $60 \%$ de ellas orientadas a textos, entre las cuales se encuentran las bases de datos referenciales y bibliográficas. Las universidades, junto con otras instituciones educativas, de investigación y gubernamentales, siguen siendo las principales consumidoras de este tipo de información, a través de suscripción. Asimismo, son varias las empresas que mantienen y comercializan un número importante de bases de datos para uso académico, por lo que puede preverse que mientras no existan otras herramientas tecnológicas para allegar resultados precisos y confiables para la investigación y la docencia, las bases de datos bibliográficas seguirán siendo un instrumento válido y necesario para la organización, almacenamiento, difusión, manejo y análisis de información académica.

\section{Discusión y conclusiones}

En la década de los setenta cuando nació Periódica, se señalaba con sentido de urgencia la necesidad de promover un mejor conocimiento de las revistas científicas y técnicas latinoamericanas, con varios retos por superar: las dificultades para obtener revistas latinoamericanas, la carencia de bibliografías nacionales sobre revistas científicas, la necesidad de crear organismos centrales de diseminación de la información, así como la carencia de bibliografías nacionales completas y de alta calidad (Maffey, 1983).
Para 2018 podemos afirmar que el panorama de las revistas científicas latinoamericanas se ha transformado radicalmente. A las compilaciones iniciadas por CLASE en 1975 y Periódica en 1978 siguieron otros esfuerzos en la región que se vieron apuntalados durante la década de los noventa con la aparición de recursos de información como Latindex y SciELO. Todos estos esfuerzos han favorecido la identificación y la obtención de información publicada en revistas latinoamericanas y por ende, han facilitado su análisis. Ciertamente, otros retos mencionados en 1983 persisten tales como la falta de presupuestos para las revistas, que es mencionado de manera recurrente en las reuniones de editores en nuestros países (Alonso et al., 2015).

La existencia de bases de datos regionales permite el análisis de un universo más amplio que el incluido, por ejemplo, en las dos bases de datos con mayor influencia en la comunidad científica internacional: Web of Science ${ }^{\circledR}$ (WoS) y Scopus $₫$. Sin embargo, y a pesar de su larga pervivencia, recursos de información como Periódica siguen siendo sub utilizados y reconocidos como fuentes de información sobre las publicaciones latinoamericanas, debido a su escasa difusión que impacta en el reconocimiento al valor de estos recursos, y también por una marcada tendencia por utilizar recursos internacionales. A pesar de esta situación, desarrollos tecnológicos innovadores como la ya mencionada fusión de recursos de Biblat e ÍnDICEs-CSIC, permitirán potenciar el impacto de este tipo de bases de datos en las comunidades académicas a las que están dirigidas.

Esencialmente, se hace necesario revalorar los esfuerzos latinoamericanos para dar difusión y promover el uso y citación de las investigaciones vertidas en las revistas latinoamericanas, ya que de lo contrario, estos recursos caerían en la categoría de "ciencia perdida" (Aguado-López, et al., 2003). Además, no debe olvidarse que los esfuerzos aquí mencionados fueron en realidad pioneros del acceso abierto a la información, antes de que se formalizara el término en 2002.

El análisis de las revistas y sus contenidos permite evidenciar cierto avance en el trabajo editorial de revistas de CTM en América Latina, tanto en términos cuantitativos como cualitativos.

El número de revistas por país refleja coincidencias con la aportación geográfica en ciencias sociales y humanas (CSH) con leves diferencias, mientras que en revistas de CTM Brasil está a la cabeza, seguido de México y Argentina, en revistas de CSH México aporta más títulos que Brasil, mientras que Colombia y Venezuela aventajan a Argentina (Reyna, 2015). 
Destaca desde luego el crecimiento de publicaciones disponibles en línea, situación que favoreció la disponibilidad de enlaces hacia los textos completos; sin embargo, éstos representan solamente el $38 \%$ del total de registros ya que Periódica ha creado la mayoría de sus registros en una época de prevalencia de la revista impresa.

El análisis por disciplinas muestra también el interés de las instituciones editoras por impulsar la investigación en temas que son de interés nacional y regional, para contribuir así a la solución de problemas que le son propios. Es por ello que no extraña que las revistas dedicadas a temas médicos, biológicos y agrícolas prevalezcan en número tal como se reflejó en el estudio de 1983, advirtiéndose un repunte en el número de revistas especializadas en ingenierías, ciencias de la tierra, así como en temas de ciencia y tecnología.

En este estudio se hizo evidente la participación mayoritaria que las instituciones educativas, de investigación, generalmente de patrocinio público, así como las asociaciones científicas sin fines de lucro tienen como impulsoras de la investigación y la edición de revistas científicas, frente a la escasa participación del sector privado. Esta es una diferencia fundamental en nuestra región, ya que en otros países, la cadena de la comunicación científica basada en revistas está más bien impulsada por entes comerciales privados.

La frecuencia de publicación en conjunto tiende a alejarse de periodicidades muy extendidas, con una tendencia hacia las intermedias que facilitan el trabajo de los editores para cumplir con el ciclo y los procesos de publicación. Sin embargo, y tal vez por las restricciones que enfrentan los equipos editoriales, las frecuencias más cortas como la mensual $(44=3.7 \%)$ u otras frecuencias $(19=1.6 \%)$ que incluyen la semanal, quincenal o continua, son todavía reducidas.

Respecto del idioma de publicación, el estudio pone de manifiesto un importante crecimiento de los artículos en inglés, en comparación con los resultados obtenidos hace 35 años. Las presiones para apuntalar la internacionalización de nuestras publicaciones seguramente han favorecido esta tendencia, pero los autores también buscan alcanzar un público más amplio y, eventualmente, obtener citas de audiencias más allá del ámbito regional. Además, muchos autores de artículos en las disciplinas de CTM, que no tienen al inglés como su lengua nativa, suelen publicar sus descubrimientos en dicho idioma dado que el inglés ha sido reconocido como la lingua franca de la ciencia (Meneghini y Packer, 2007).

Los datos de Periódica también evidencian que las revistas latinoamericanas de CTM pueden ser vehículo internacional de publicación para autores de otras latitudes. Esto se refleja en las adscripciones ubicadas en 140 países diferentes que han sido codificadas a lo largo del tiempo. Si bien las instituciones latinoamericanas constituyen la mayoría, llama la atención la notable contribución de Estados Unidos y España, cada uno con más de 11,000 adscripciones, por lo que ocupan un lugar entre los 10 países con más adscripciones en revistas latinoamericanas de CTM, superando a varios países de la región.

La diversidad geográfica resulta relevante ya que se tienen registrados autores de instituciones ubicadas en el Medio Oriente, Sudeste Asiático o en África, que encuentran en las revistas latinoamericanas un vehículo de difusión de sus trabajos que para ellos resultan ser "internacionales". Se encontraron adscripciones ubicadas en países como Arabia Saudita, Burkina Faso, Corea del Sur, Egipto, Filipinas, Ghana, Kenia, Malasia, Turquía o Papúa Nueva Guinea, por citar solo algunos que han sido compilados en la base de datos.

Finalmente, entre los desafíos que enfrenta $\mathrm{Pe}$ riódica en su futuro mediato se encuentra la necesidad de contar con un software manejador de bases de datos que cumpla mejor con los requerimientos de usuarios y administradores. $Y$ aunque la tendencia es hacia la edición de revistas en formato electrónico, subsiste el interés por digitalizar la colección de revistas impresas de la Hemeroteca Latinoamericana, para dar acceso cabal a esos materiales de carácter multidisciplinario que durante varias décadas fueron publicados únicamente en formato impreso.

Se puede mencionar que pocos emprendimientos han logrado tener la pervivencia de servicios de información como Periódica en América Latina. Después de cuatro décadas de existencia, Periódica se encuentra vigente como una fuente de indexación buscada por los editores, así como fuente de documentación para académicos, investigadores y estudiantes.

\section{Notas}

Agradecimiento a Guadalupe Argüello Mendoza, del Departamento de Bibliografía Latinoamericana de la DGB-UNAM, por los datos fuente de las revistas indexadas en Periódica.

\section{Referencias}

Aguado-López, Eduardo; Sandoval-Forero, Eduardo; Chávez-Ávila, Salvador. (2003). La ciencia perdida y las nuevas tecnologías de divulgación del conocimiento: el proyecto Redalyc. // Revista Educación y Ciencia. 7:13 (2003).

Alonso-Gamboa, José Octavio (1998). Acceso a revistas latinoamericanas en Internet. Una opción a través de las bases de datos CLASE y Periódica. // Ciência da Informação. $27: 1$ (1998). 
Alonso Gamboa, José Octavio; Arana Mendoza, Celia; Reyna Espinosa, Felipe Rafael; Sánchez Pereyra, Antonio (2012). Manual de indización para las bases de datos CLASE y Periódica. México: UNAM, 2012. 107 p. ISBN 978-607-02-2906-0.

Alonso Gamboa, José Octavio; Reyna Espinosa, Felipe Rafael; Sánchez Islas, Liliana Andrea (2015). Características y calidad editorial de las revistas científicas mexicanas: la aportación de Latindex. // Ciência da Informação. 44:2 (2015)

Cetto Kramis, Ana María; Alonso Gamboa, José Octavio (1998). Scientific periodicals in Latin America and the Caribbean: a global perspective. // Interciencia. 23:2 (2018).

Chong-Carrillo, Olimpia; Vega-Villasante, Fernando; Maciel, Cristiana; Álvarez-González, Carlos Alfonso; MichánAguirre, Layla; Arencibia-Jorge, Ricardo (2018). Scientometrics of the genus Macrobrachium by using the regional PERIODICA database. // Latin American Journal of Aquatic Research. 46:3 (2018). DOI: 10.3856/vol46-issue3-fulltext-11

Giménez-Toledo, Elea; Rodríguez-Yunta, Luis (2012). ¿Por qué nadie habla ya de las bases de datos bibliográficas "clásicas"?. // Anuario ThinkEPI. Vol. 6 (2012).

Maffey, L.; Arana Mendoza, C.; Deschamps, I.; Sosa, V. (1983). "Periódica" un índice de revistas latinoamericanas en ciencia y tecnología. // Revista española de documentación científica. 6:2 (1983).

Meneghini, Rogerio; Packer, Abel (2007). Is there Science beyond English?. // EMBO Reports. 8:2 (2007). DOI: 10.1038/sj.embor.7400906

Michán, Layla; Russell, Jane Margaret; Sánchez Pereyra, Antonio; Llorens Cruset, Antonia; López Beltrán, Carlos (2008). Análisis de la sistemática actual en Latinoamérica. // Interciencia. 33:10 (2008).

Reyna Espinosa, Felipe Rafael (2015). CLASE: perfil de una base de datos bibliográfica. // Biblioteca Universitaria. 18:2 (2015).

\section{Apéndice: Sitios web citados}

Biblat - https://biblat.unam.mx/es

Gale - https://www.cengage.com/

Latindex - http://www.latindex.org

Base de datos Periódica - http://periódica.unam.mx

Enviado: 2018-05-18. Segunda versión: 2018-09-19 Aceptado: 2018-10-10. 